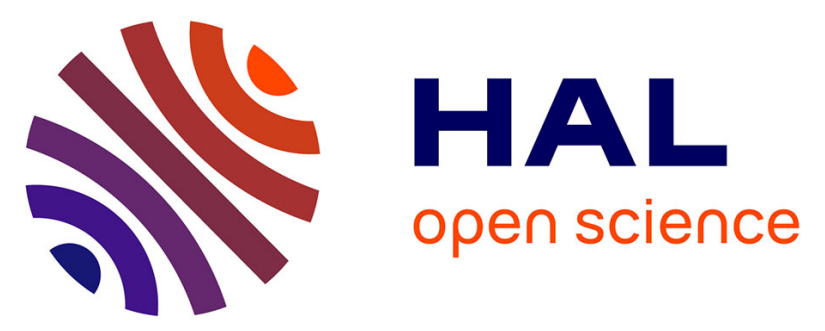

\title{
Dynamic Force/Position Modeling of a one-DOF Smart Piezoelectric Micro-Finger with Sensorized End-Effector.
}

Bilal Komati, Cédric Clévy, Micky Rakotondrabe, Philippe Lutz

\section{To cite this version:}

Bilal Komati, Cédric Clévy, Micky Rakotondrabe, Philippe Lutz. Dynamic Force/Position Modeling of a one-DOF Smart Piezoelectric Micro-Finger with Sensorized End-Effector.. IEEE/ASME International Conference on Advanced Intelligent Mechatronics, AIM'2014., Jan 2014, France. pp.1-6. hal-01026535

\section{HAL Id: hal-01026535 \\ https://hal.science/hal-01026535}

Submitted on 21 Jul 2014

HAL is a multi-disciplinary open access archive for the deposit and dissemination of scientific research documents, whether they are published or not. The documents may come from teaching and research institutions in France or abroad, or from public or private research centers.
L'archive ouverte pluridisciplinaire HAL, est destinée au dépôt et à la diffusion de documents scientifiques de niveau recherche, publiés ou non, émanant des établissements d'enseignement et de recherche français ou étrangers, des laboratoires publics ou privés. 


\title{
Dynamic Force/Position Modeling of a one-DOF Smart Piezoelectric Micro-Finger with Sensorized End-Effector
}

\author{
Bilal Komati, Cédric Clévy, Micky Rakotondrabe and Philippe Lutz
}

\begin{abstract}
In this paper, a generic microscale system is studied where a smart microsystem composed of an active based material actuator, sensorized structure and transformation system is studied. This problem is important at the microscale because it offers a force measurement of the applied force by the actuator to a flexible environment which enables to understand the interaction between the complete smart microsystem and the environment and to design and control the interaction between the system and the environment. A special case where a sensorized end-effector is fixed on the tip of a piezoelectric actuator is detailed. Integrating a sensorized end-effector influences the behavior of the smart microfinger and is not studied in recent works.

The complete finger, which is called in this paper smart finger, consists of a piezoelectric actuator, an end-effector and a novel piezoresistive force sensor. A complete model is developed for generating both force and displacement at the finger's tip while interaction with a flexible environment. A nonlinear model of the piezoelectric actuator is considered and a complete model is developed taking into account the frequency dependent hysteresis of the piezoelectric actuator. The model of the hysteresis is based on the Bouc-Wen method which simplifies the parameter estimation. The complete dynamic force/position model of the finger is validated experimentally with small errors (less than $10 \%$ ).
\end{abstract}

\section{INTRODUCTION}

Piezoelectric materials are commonly used for the actuation of microsystems and microrobots. Their use is due to their high resolution, high speed, low response time and their ability to produce large forces. They are widely used for the development of microgrippers [1], robotic platforms [2], nanopositioning [3] for examples. However, the problem of the piezoelectric materials are the presence of nonlinearities which increases the difficulty of modeling and control of such systems. The nonlinearities are notably manifested by the hysteresis and the creep. The modeling and the control of the hysteresis and of the creep have raised many works, including linear approximation and linearization [4], algebraic or differential nonlinear modeling [5], [6], feedforward control [7], [8] and feedback control [9] [10], [11].

Most of the developed models study the model of voltage/displacement of the piezoelectric actuator. Some other works, study the voltage/force/displacement model of the piezoelectric actuator [9], [12]. These models are limited for the piezoelectric actuator itself and do not study the problem of adding an end-effector to the piezoelectric actuator which modifies the complete model of the finger. However, for

The authors are with FEMTO-ST Institute, AS2M department,Université de Franche-Comté/CNRS/ENSMM, 24 rue Alain Savary, 25000 Besancon, France (e-mail: bilal.komati@femto-st.fr; cclevy@femto-st.fr; mrakoton@femto-st.fr; philippe.lutz@femto-st.fr). many applications, it is important to integrate force measurement into a piezoelectric actuator in order to develop the complete model of the piezoelectric actuator, determine the contact, study the interaction between the actuator and the environment and control precisely the interaction. Most of the force sensing techniques used for the piezoelectric actuators are the self-sensing [13], force estimation using models [14] and vision based force sensors [15]. However, the integration of calibrated force sensors is of great interest for precise dynamic measurement for many applications.

In this paper, a generic microscale problem is studied where a classic piezoelectric actuator is used to interact with an environment. However, in order to model the interaction between the actuator and the environment, a sensorized endeffector is fixed on the tip of the piezoelectric actuator. The end-effector is composed of a rigid silicon part and an novel piezoresistive force sensor presented in [16]. This generic problem can be extended to other examples of microsystems where actuator and force sensors are integrated. The complete finger composed of a piezoelectric actuator with a sensorized end-effector is called in this paper Smart Finger. Unfortunately, the complete model of such a smart finger is not studied in literature to the knowledge of the authors.

The first objective of the paper is to develop a precise position/force dynamic nonlinear model of the smart finger which enables to design and control such a smart finger interaction with a flexible environment. The model is developed taking into consideration the effect of adding a sensorized end-effector to the piezoelectric actuator which influences the existing force/position models to the piezoelectric actuator without an end-effector. The second objective of the paper is to experimentally validate the developed model.

The paper is organized as follows. The piezoelectric actuator nonlinear dynamic model is revised in section II. The model of the smart finger at the sensorized end-effector's tip is detailed in section III. In section IV, an experimental setup is proposed to validate the model of the smart finger. Section V presents the experimental results to validate the model. Section VI concludes the paper.

\section{Piezoelectric Actuator Model}

A generic microrobotic scenario is considered in this paper. Consider that the smart finger comes into contact with a flexible environment. The environment is modeled as a mass-spring-damper system as it was done in [17]. The complete system is shown in Figure 1 where four cases are considered: case of the no contact no voltage are applied (Figure 1-(a)), case of an applied voltage without contact 
(Figure 1-(b)), case of applied voltage and just at contact with a zero force (Figure 1-(c)) and case of both voltage and contact applied (Figure 1-(d)). In this figure, $A$ and $B$ represent respectively the actuator's tip A and the endeffector's tip B. $y_{A}$ and $y_{B}$ represent respectively the position of the actuator's tip A and the end-effector's tip B at time $t$. $y_{e}$ represents the location of the environment without any deformation when no force is applied to the environment $\left(y_{e}=y_{B}\right.$ just at contact when the applied force is null). $y=y_{B}$ represents the position of the environment and the end-effector tip B when a contact is established between the end-effector and the environment. $\alpha_{A}$ is the angle at the actuator tip A. $L$ is the length of the end-effector. $m_{e}, d_{e}$ and $k_{e}$ are respectively the mass, the damping and the stiffness of the environment.

In order to determine the complete model for the smart finger, the piezoelectric actuator's voltage/force/position nonlinear model is revised in this section and the complete model of the smart finger is then detailed in section III. The aim is to develop a simple dynamic nonlinear model in order to facilitate the identification process. The inputs of the model of the piezoelectric actuator are the voltage applied on the electrodes of the piezoelectric actuator, $U$, and the forces and the torque applied to the actuator's tip A (as considered in literature) and the outputs are the displacement at the actuator's tip A and the angle at the tip of the actuator $\alpha_{A}$ (see Figure 1).
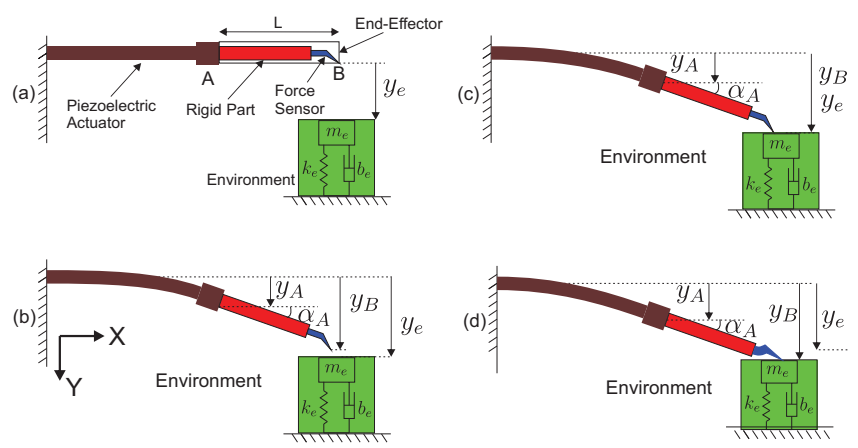

Fig. 1. Complete scheme of the smart finger and the environment: (a) without contact and without any applied voltage, (b) without contact and with an applied voltage, (c) just at contact $\left(F_{e}=0\right)$ and with an applied voltage and (d) with contact $\left(F_{e} \neq 0\right)$ and with an applied voltage.

Using [12], [18], a piezoelectric actuator could be modeled in the time domain as follows:

$$
\left\{\begin{array}{l}
m \ddot{y}_{A}+b \dot{y}_{A}+k y_{A}=d u-\Gamma-f_{A}-\frac{3}{2 L_{a}} M_{A} \\
m \ddot{\alpha}_{A}+b \dot{\alpha}_{A}+k \alpha_{A}=\frac{2}{L_{a}}(d u-\Gamma)-\frac{3}{2 L_{a}} f_{A}-\frac{3}{L_{a}^{2}} M_{A}
\end{array}\right.
$$

where parameters $m, b$ and $k$ are respectively the mass, the damping and the stiffness of piezoelectric actuator; $d$ is the piezoelectric constant, $L_{a}$ is the length of the actuator, $u=$ $u(t)$ is the input voltage, $y_{A}=y_{A}(t)$ is the displacement of the actuator's tip A according to the $Y$ axis and $\alpha_{A}=\alpha_{A}(t)$ is the angle between the actuator's tip A and the $X$ axis (see Figure 1), $f_{A}=f_{A}(t)$ and $M_{A}=M_{A}(t)$ are respectively the force and the torque applied at the actuator's tip A and $\Gamma=\Gamma(t, u, f)$ is an operator to represent the nonlinearities of the piezoelectric actuator which consist of rate-dependent hysteresis and creep. The variable time $t$ is simplified from the equations for the simplicity of the writing.

In the rest of the paper, the frequency domain is used for the equations where the Laplace operator, $s$ will be used instead of the time domain. Knowing that [9]:

$$
\Gamma(U, s)=H(U, s)+C_{r}(U, s)
$$

where $H(U, s)$ and $C_{r}(U, s)$ are respectively the hysteresis and the creep of the piezoelectric actuator. According to (2), the two nonlinearity phenomenas could be decoupled and each could be treated in an independent way. The effect of the creep is neglected in this paper.

The hysteresis of piezoelectric material is called ratedependent hysteresis because the hysteresis is function of the frequency or the rate of the input of the system. An approximate model of the rate-dependent hysteresis have parameters which are also rate-dependent. This will increase the complexity of the model. This problem is simplified in [9] where the authors proposed to model the dynamical hysteresis of the piezoelectric actuator, $H(U, s)$, by a static hysteresis, $H_{S}(U)$, followed by a linear dynamical part, $D(s)$, as shown in Figure 2. $D(s)$ is a second order transfer function which represents the dynamics of the actuator. In Figure 2,

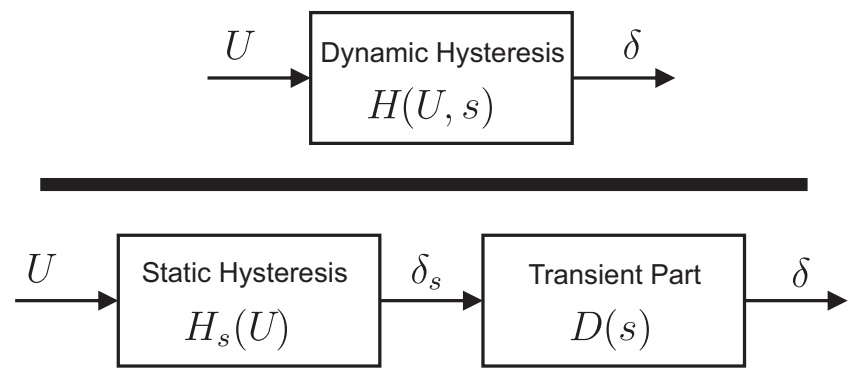

Fig. 2. Dynamic hysteresis could be decomposed in a static part followed by a dynamical part.

$U$ is the voltage applied to the piezoelectric actuator, $\delta$ is the displacement of the actuator tip, $\delta_{s}$ is the static displacement which is the output of the Bouc-Wen static hysteresis model, $H(U, s)$ is the rate-dependent hysteresis which depends on the voltage, $U$, and the frequency which is written as the Laplace operator, $s=j 2 \pi f$, and $H_{s}(U)$ is the static hysteresis determined by the Bouc-Wen model.

The dynamical hysteresis of the piezoelectric actuator is determined using the following steps, [8]:

1) The transient part of the piezoelectric actuator, $D(s)$, is identified. The dynamical part could be considered independent of the amplitude of the input voltage $U$. It is determined by applying several steps of different amplitudes and then the dynamical response could be approximated by a second order transfer function as is commonly used. 
2) We apply a sine voltage with a low frequency $(0.1 \mathrm{~Hz})$ and identify the Bouc-Wen parameters to minimize the error between the experimental result of the displacement of the actuator and the Bouc-Wen model for the displacement of the actuator. Using this method we determine $H_{s}(U)$.

3) The dynamical hysteresis of the piezoelectric actuator is determined, using the two previous steps, by the relation $H(U, s)=H_{s}(U) \cdot D(s)$.

Finally, using the Bouc-Wen model presented in [19] and using the Laplace transform of (1), the adopted model for the displacement at the tip of the piezoelectric actuator is given, in the frequency domain, by the following:

$$
\left\{\begin{array}{l}
y_{A}(s)=\left[d_{p} U-H_{s}-s_{p} F_{A}-\frac{3}{2 L_{a}} s_{p} M_{A}\right] D(s) \\
\alpha_{A}(s)=\left[\frac{2}{L_{a}}\left(d_{p} U-H_{s}\right)-\frac{3}{2 L_{a}} s_{p} F_{A}-\frac{3}{L_{a}^{2}} s_{p} M_{A}\right] D(s) \\
\dot{H}_{s}=\alpha \dot{U}-\beta|\dot{U}| H_{s}-\gamma \dot{U}\left|H_{s}\right|
\end{array}\right.
$$

where

- $y_{A}$ is the displacement of the piezoelectric actuator's tip A along the $Y$ axis,

- $\alpha_{A}$ is the angle between the actuator's tip A and the $X$ axis,

- $d_{p}=\frac{d}{k}$ is the piezoelectric constant and $s_{p}=\frac{1}{k}$ is the elastic constant,

- $F_{A}$ is an external force applied at the actuator's tip A,

- $M_{A}$ is an external torque applied at the actuator's tip A,

- $U$ is the applied voltage to the piezoelectric actuator,

- $H_{s}$ is an internal variable to represent the hysteresis,

- $D(s)$ is a transfer function to represent the dynamics of the actuator,

- $\alpha$ is a parameter which determines the amplitude of the hysteresis,

- $\beta$ and $\gamma$ are parameters which determine the shape of the hysteresis.

Second order transfer functions are commonly used to model the dynamics of a piezoelectric actuator [12], [9]. $D(s)$ is then considered as a second order transfer function with a static gain $1(D(0)=1)$. It can be written as follows:

$$
D(s)=\frac{k}{m s^{2}+b s+k}
$$

\section{MODEL OF THE SMART FINGER AT THE END-EFFECTOR TIP}

The aim of this section is to develop the model of the complete smart finger with and without contact with the environment. The inputs of the smart finger in this case are the voltage applied on the piezoelectric actuator electrodes, $U$, and the distance between the equilibrium position of the environment without any contact and the $X$ axis, $y_{e}$, while the outputs of the system are the position of the sensorized end-effector's tip B and the interaction force between the end-effector and the environment, $F_{e}$.

The model of smart finger is developed using the model developed in (3) of the piezoelectric actuator where a force and a torque is applied at the tip A of the piezoelectric actuator $\left(f_{A}\right.$ and $\left.M_{A}\right)$. Then, However, in the case of the paper, the force will be applied at the end-effector's tip B. Thus, the effect of applying a force at the end-effector's tip $\mathrm{B}$ is studied and the relation between the force applied at the tip B and the reaction forces and torque at the tip A is determined. Finally, the complete model of the smart finger when a force is applied at tip B is deduced for the non contact and contact scenarios. One unified model is developed and derived from the two models.

Starting with the case where a force is applied by the environment at the force sensor's tip B, a reaction force $F_{A}$ and a reaction torque $M_{A}$ exist on the actuator's tip A, which can be written as follows:

$$
\left\{\begin{aligned}
F_{A} & =-F_{s}+m_{s} \ddot{y}_{B}=F_{e}+m_{s} \ddot{y}_{B} \\
M_{A} & =-L F_{s}=L F_{e}
\end{aligned}\right.
$$

where $F_{e}=F_{\text {end-effector } \rightarrow \text { environment }}$ is the force applied by the end-effector to the environment and $F_{s}=-f_{e}=$ $F_{\text {environment } \rightarrow \text { end-effector }}$ is the force applied by the environment to the end-effector when a contact exists; $m_{s}$ is the mass of the force sensor. The mass of the force sensor is neglected because it is small compared to the mass of the actuator $\left(m_{s}=0\right)$.

As the force sensor is also flexible, it can be modeled as a mass-spring-damper system where the mass is neglected and then the force sensor is modeled as a spring-damper. On a force $F_{S}$ is applied at the force sensor's tip B, the force sensor deforms and the displacement of the force sensor is $\delta=y_{B}-y_{B}^{o}$ where $y_{B}^{o}$ is the supposed position of the force sensor's tip B without considering the deformation of the force sensor. The relation between the $F_{S}, y_{B}$ and $y_{B}^{o}$ is given, in the time domain, as follows:

$$
F_{s}=-F_{e}=b_{s}\left(\dot{y}_{B}-\dot{y}_{B}^{o}\right)+k_{s}\left(y_{B}-y_{B}^{o}\right)
$$

where $b_{s}$ and $k_{s}$ are respectively the damping and the stiffness of the force sensor and its mass is neglected. $y_{B}^{o}$ is given as follows:

$$
y_{B}^{o}=y_{A}+L \sin \alpha_{A}
$$

Since $\alpha_{A}$ is supposed to be small $\sin \alpha_{A} \approx \alpha_{A}$ and using (3), (5), and (7), $Y_{B}^{o}$ is given as follows:

$$
\begin{aligned}
Y_{B}^{o}(s)= & {\left[\left(1+\frac{2 L}{L_{a}}\right)\left(d_{p} U-H_{s}\right)-\right.} \\
& \left.\left(1+\frac{3 L}{2 L_{a}}+\frac{3 L^{2}}{L_{a}^{2}}\right) s_{p} F_{e}\right] D(s)
\end{aligned}
$$

Using (8) and the Laplace transform of (6), the displacement at the end-effector's tip B is given as follows:

$$
\begin{aligned}
Y_{B}(s)= & \frac{L_{u} k}{m s^{2}+b s+k}\left(d_{p} U-H_{s}\right)- \\
& \frac{m s^{2}+\left(b+b_{s} L_{f}\right) s+k+k_{s} L_{f}}{\left(b_{s} s+k_{s}\right)\left(m s^{2}+b s+k\right)} F_{e}
\end{aligned}
$$

where $L_{u}=1+\frac{2 L}{L_{a}}$ and $L_{f}=1+\frac{3 L}{L_{a}}+\frac{3 L^{2}}{L_{a}^{2}}$ In addition, the force applied by the end-effector on the environment is given 
by:

$$
f_{e}=\left\{\begin{array}{lll}
m_{e} \ddot{y}_{B}+b_{e} \dot{y}_{B}+k_{e}\left(y_{B}-y_{e}\right) & \text { if } & y_{B} \geq y_{e} \\
0 & \text { if } & y_{B}<y_{e}
\end{array}\right.
$$

where $m_{e}, b_{e}$ and $k_{e}$ are respectively the mass, damping and stiffness of the environment. Using (9) and the Laplace transform of (10), the following could be deduced:

$$
F_{e}(s)=\left\{\begin{array}{lll}
G_{u}(s)\left[d_{p} U-H_{s}-G_{e}(s) Y_{e}\right] & \text { if } \quad y_{B} \geq y_{e} \\
0 & \text { if } y_{B}<y_{e}
\end{array}\right.
$$

where:

$$
\left\{\begin{array}{l}
G_{u}(s)=\frac{L_{u} k\left(m_{e} s^{2}+b_{e} s+k_{e}\right)\left(b_{s} s+k_{s}\right)}{H(s)} \\
G_{e}(s)=\frac{k_{e}\left(m s^{2}+b s+k\right)}{L_{u} k\left(m_{e} s^{2}+b_{e} s+k_{e}\right)}
\end{array}\right.
$$

where

$$
\begin{aligned}
H(s)= & m_{e} s^{4}+\left[m b_{s}+m b_{e}+m_{e}\left(b+L_{f} b_{s}\right)\right] s^{3}+ \\
& {\left[m k_{s}+b b_{s}+m k_{e}+m_{e}\left(k+L_{f} k_{s}\right)+\right.} \\
& \left.b_{e}\left(b+L_{f} b_{s}\right)\right] s^{2}+ \\
& {\left[b k_{s}+k b_{s}+b_{e}\left(k+L_{f} k_{s}\right)+k_{e}\left(b+L_{f} b_{s}\right)\right] s+} \\
& k k_{s}+k_{e}\left(k+L_{f} k_{s}\right)
\end{aligned}
$$

Using (9) and (11), the final model at the end-effector's tip $B$ could be written as follows:

$$
\left\{\begin{aligned}
Y_{B}= & {\left[\left(1+\frac{2 L}{L_{a}}\right)\left(d_{p} U-H_{s}\right)-\right.} \\
& \left.\left(1+\frac{3 L}{2 L_{a}}+\frac{3 L^{2}}{L_{a}^{2}}\right) s_{p} F_{e}\right] D^{\prime}(s) \\
F_{e}= & \left\{\begin{array}{lll}
G_{u}(s)\left[d_{p} U-H_{s}-G_{e}(s) Y_{e}\right] & \text { if } y_{B} \geq y_{e} \\
0 & \text { if } y_{B}<y_{e}
\end{array}\right. \\
\dot{H}_{s}= & \alpha \dot{U}-\beta|\dot{U}| H_{s}-\gamma \dot{U}\left|H_{s}\right|
\end{aligned}\right.
$$

The complete model of the smart finger can be represented by the scheme in figure 3 where the inputs of the model are $U$ and $y_{e}$ and the outputs are $y_{B}$ and $F_{e}$.

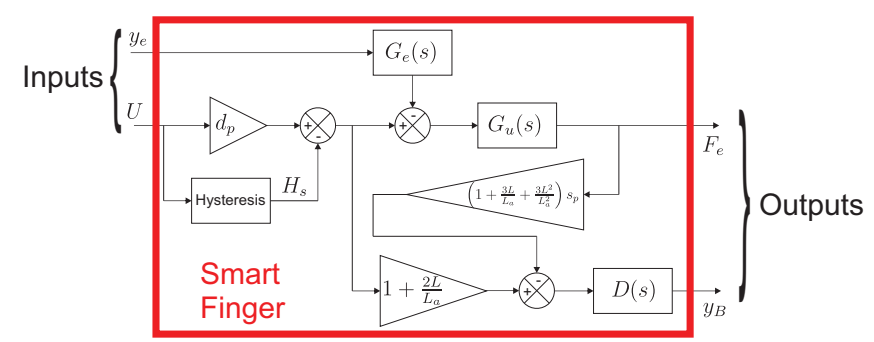

Fig. 3. Bloc diagram showing the model of the complete smart finger in interaction with a flexible environment.

\section{EXPERIMENTAL SETUP}

The experimental setup used in this paper, in order to validate the developed model, is composed of a smart finger and displacement and force sensors. The smart finger is composed of a duo bimorph piezoelectric actuator presented in [1] and a novel piezoresistive force sensor presented in [16]. Two Keyence laser sensors are used to measure the displacement of the actuator's tip A and the end-effector's tip B. A force sensor from FemtoTools, with a sensing range of $10 \mathrm{mN}$, is fixed on a XY positioning stage from PiezoSystemJena, PXY D12 with a displacement range of $200 \mu \mathrm{m}$. The force sensor comprises a probe of $3 \mathrm{~mm}$ of length and $50 \mu \mathrm{m}$ of thickness, that moves along its main direction ( $Y$ according to Figure 4) once a force is applied at its tip. The XY positioning is used to move the FemtoTools force sensor towards the piezoresistive force sensor to calibrate the force sensor and to simulate a contact between the smart finger and the force sensor. The whole system is controlled using a dSpace1104 with a sampling frequency of $10 \mathrm{kHz}$. The experimental setup is shown in Figure 4.

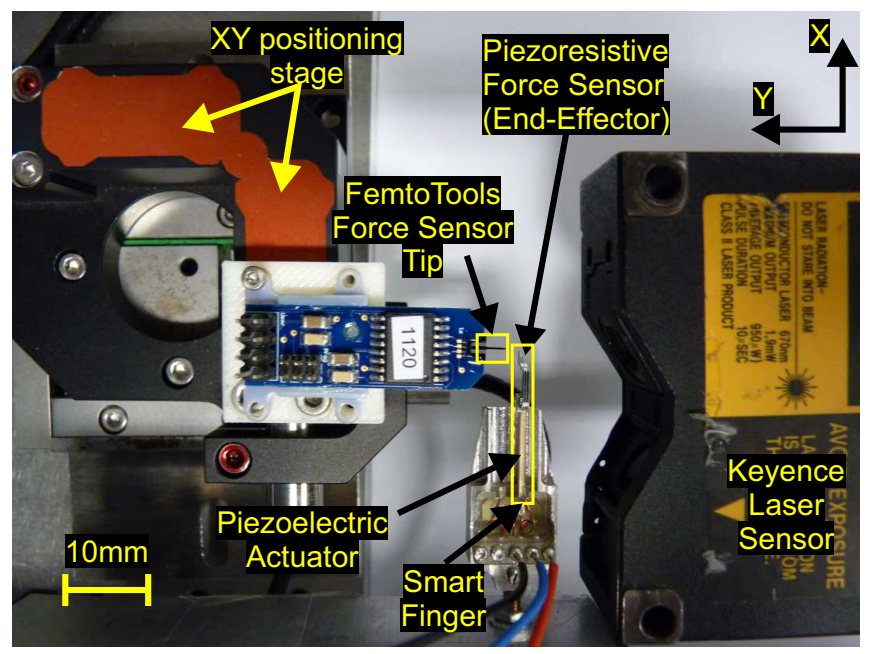

Fig. 4. The whole experimental setup used in this paper including the smart finger, the XY positioning stage, a Keyence laser sensor and the FemtoTools force sensor.

\section{EXPERIMENTAL STUDIES}

In this section, the presented model in section III is verified experimentally. The validation of the model is performed in three steps. First, the model of the displacement/voltage is determined without any contact between the smart finger and the environment. Then, the model of force/displacement is determined without any applied voltage. Finally, the complete model (displacement/force/voltage), defined in (13), is tested by applying both voltage and contact to the smart finger. The identified parameters are summarized in Table I.

\section{A. Displacement/Voltage Model of the Smart Finger}

In this section, no force is applied at the tip of the smart finger. Two Keyence sensors are used to measure the displacement at points $\mathrm{A}$ and $\mathrm{B}$ of the actuator and end-effector respectively. The experiments are done in the following steps:

1) the parameters of the actuator, $d_{p}, \alpha, \beta$ and $\gamma$, defined in (3) are identified by applying a sine wave with a frequency of $0.1 \mathrm{~Hz}$ (to ignore the dynamic part $D(s)$ ) without any force and using the nonlinear least square 
method in order to fit the experimental results with the model. A comparison between the model and the experiments after the parameter estimation is shown in Figure 5 for three different amplitudes of the sine waves $(30 \mathrm{~V}, 60 \mathrm{~V}$ and $100 \mathrm{~V})$,

2) the dynamic part, $D(s)$, of piezoelectric actuator is determined by applying several steps with different amplitudes and determining the second order transfer function which fits the best to the experimental data. The dynamic response of the piezoelectric actuator is independent of the voltage amplitude. The parameters $\frac{m}{k}$ and $\frac{b}{k}$ of (4) are then determined in Table I,

3) the model of $y_{B}$ defined in (13) is tested without contact and at different frequencies of sine waves. The results are shown in Figure 6.

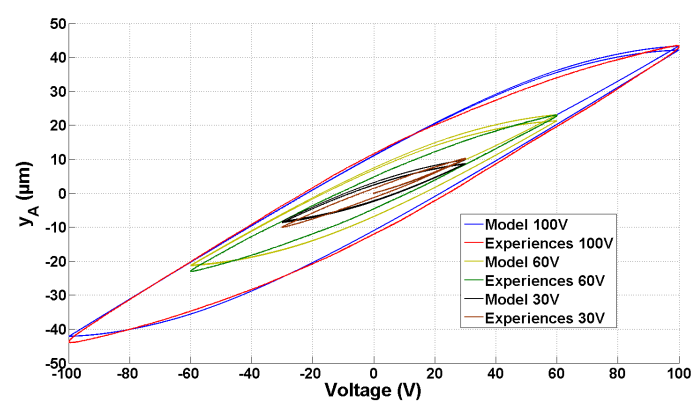

Fig. 5. Comparison between the Bouc-Wen static hysteresis model and experimental results for many sine voltages with three different amplitudes $30 \mathrm{~V}, 60 \mathrm{~V}$ and $100 \mathrm{~V}$ at a frequency $0.1 \mathrm{~Hz}$.
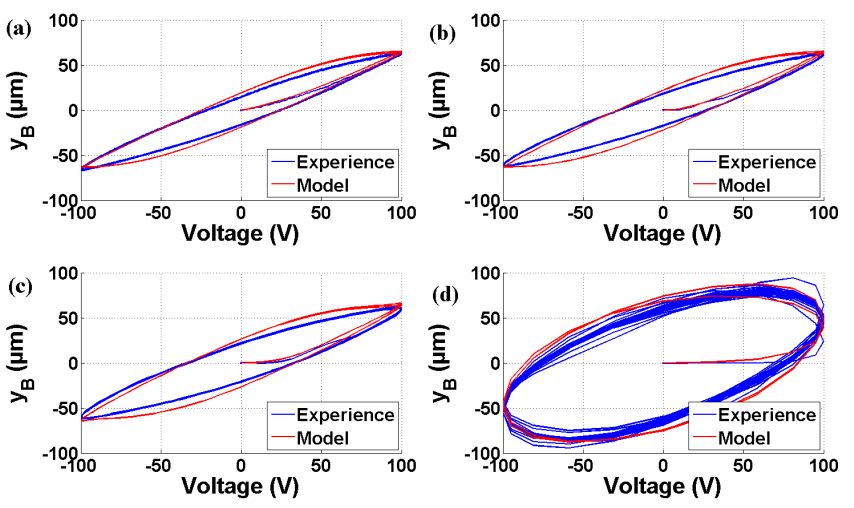

Fig. 6. Comparison between the displacement model of the end-effector tip $\mathrm{B} y_{B}$ given in (13) and experimental results for many sine voltages with an amplitude of $100 \mathrm{~V}$ and with different frequencies where no force is applied: (a) $10 \mathrm{~Hz}$, (b) $50 \mathrm{~Hz}$, (c) $100 \mathrm{~Hz}$ and (d) $500 \mathrm{~Hz}$.

Small errors (less than 10\%) between the model and the experimental result exist on the displacement/voltage model due to the model of Bouc-Wen used where the dynamic hysteresis is estimated using the static hysteresis determined by the Bouc-Wen method, $H_{S}(U)$, and the transient part of the actuator, $D(s)$ and the dynamic hysteresis is identified by $H(U, s)=H_{s}(U) \cdot D(s)$

\section{B. Force/Displacement Model of the Smart Finger}

After validating the displacement/voltage model of the smart finger, the electrodes of the piezoelectric actuator are short circuited and a force is applied first at tip A and second at tip B in order to test the force/displacement model of the smart finger. The experiments are done in the following steps:

1) parameters $s_{p}$ and $k$ are determined by applying a force by moving the FemtoTools sensor towards tip A and measuring the displacement at tips $\mathrm{A}$ and $\mathrm{B}$,

2) a force is applied at tip B and the models defined in (3) and (13) are tested without voltage (eliminating parameters $U$ and $H_{s}$ ). The comparison between the models and the experimental results is shown in Figure 7 where the model fits the experimental results with an error less than $2 \%$.
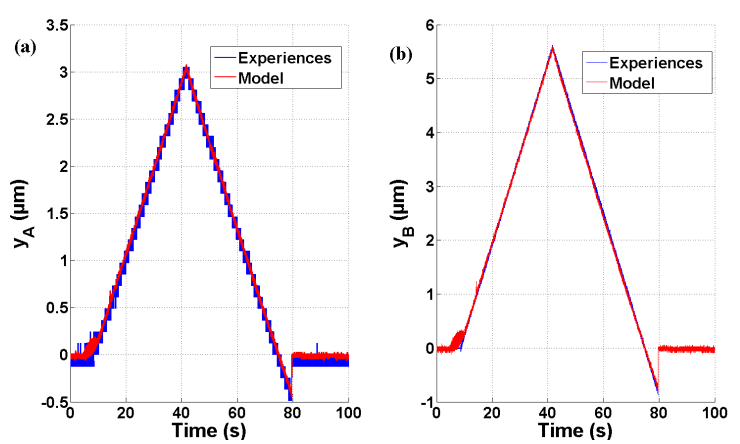

Fig. 7. Comparison between the displacement model at tips $\mathrm{A}$ and B, $y_{A}$ and $y_{B}$, given in (13) and (3) and experimental results when a force is applied at the end-effector's tip B in static and with a short circuit on the electrodes of the actuator: (a) comparison at actuator's tip A, $y_{A}$, and (b) comparison at end-effector's tip B, $y_{B}$

\section{Complete Force/Displacement/Voltage Model of the Smart Finger}

In this section, after validation the displacement/voltage and force/displacement, the two models are combined to test the complete displacement/force/voltage. Sine waves are applied to the actuator's electrodes. The smart finger starts deforming and approaches the environment until entering in contact with the environment and the force starts to increase. Then, the smart finger returns back and separate the contact. The action is repeated many times. The models of $y_{B}$ and $F_{e}$ given in (13) are compared to the experimental results in Figure 8. The experimental results fit to the model with errors less than $10 \%$.

All the experimental results fit with the developed model (13) with errors less than $10 \%$. Thus, the developed model in (13) is validated. The identified parameters of the smart finger are shown in Table I.

\section{CONCLUSION}

In this paper, a generic microscale problem is studied where a sensorized end-effector is fixed on the tip of a piezoelectric actuator. This problem is important at the microscale because it offers a force measurement of the applied force by the actuator to a flexible environment which enables to understand the interaction between the complete finger (actuator + sensorized end-effector) and to design and control the interaction between the finger and the environment. The 


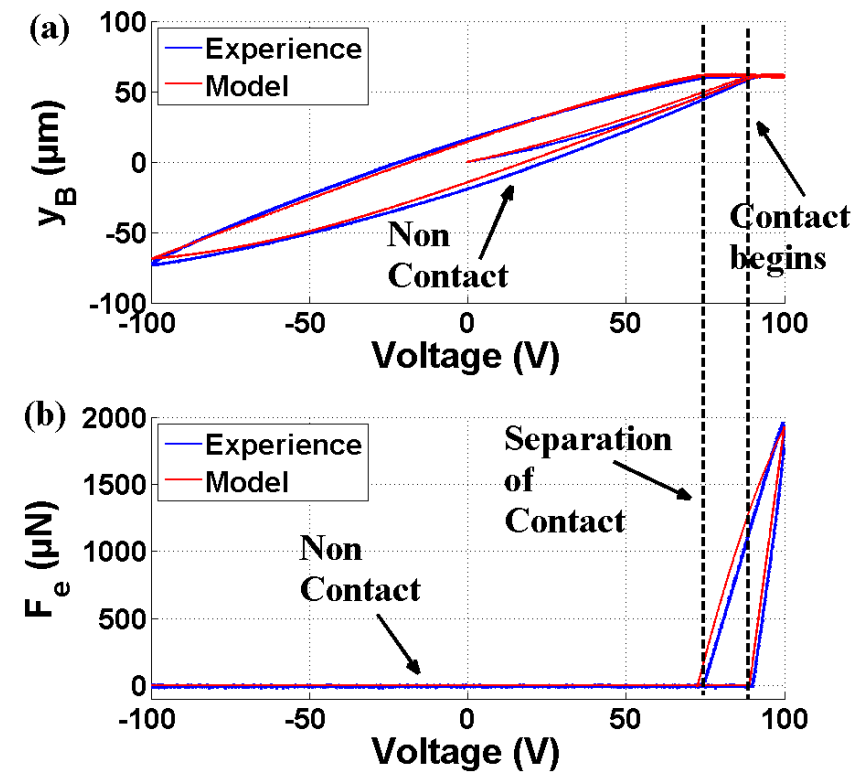

Fig. 8. Comparison between the model of the force, given in (13), applied by the smart finger on the environment and the measured force.

\begin{tabular}{|c|c|}
\hline Parameter & Identified Values \\
\hline$d_{p}$ & $0.54 \mu \mathrm{m} / \mathrm{V}$ \\
\hline$s_{p}$ & $3.57 \times 10^{-4}$ \\
\hline$\alpha$ & 0.316 \\
\hline$\beta$ & 0.019 \\
\hline$\gamma$ & 0.0033 \\
\hline$m$ & $1.347 \times 10^{-4}$ \\
\hline$b$ & 0.469 \\
\hline$k$ & 2800 \\
\hline$L_{a}$ & $13.5 \mathrm{~mm}$ \\
\hline$L$ & $8 \mathrm{~mm}$ \\
\hline$k_{s}$ & $130 \mathrm{~N} / \mathrm{m}$ \\
\hline
\end{tabular}

TABLE I

THE IDENTIFIED PARAMETERS USED IN THIS PAPER

effect of adding a sensorized end-effector influences the behavior of the actuator and is not studied in recent works.

The complete finger, which is called in this paper smart finger, consists of a piezoelectric actuator, an end-effector and a novel piezoresistive force sensor. A complete model is developed for both force and displacement at the finger's tip while interaction with a flexible environment. A nonlinear model of the piezoelectric actuator is considered and a complete model is developed taking into account the frequency dependent hysteresis of the piezoelectric actuator. The model of the hysteresis is based on the Bouc-Wen method which simplifies the parameter estimation. The complete model of the finger is validated experimentally with small errors (less than 10\%). These errors are due to the approach of modeling the rate-dependent hysteresis which simplifies the identification of the parameters and the implementation of the method. These errors can be reduced by using more complex and advanced models of the rate-dependent hysteresis which is not the objective of the paper.
This paper offers a complete theoretical method for modeling such systems where this method can be used to model other types of actuators and actuated MEMS structures.

\section{ACKNOWLEDGMENT}

These works have been partially funded by the FrancheComte region, the Labex ACTION project (contract "ANR11-LABX-01-01") and the French RENATECH network through its FEMTO-ST technological facility.

\section{REFERENCES}

[1] P. de Lit, J. Agnus, C. Clévy, and N. Chaillet, "A four-degree-offreedom microprehensile microrobot on chip," Assembly and Automation, vol. 24, no. 1, pp. 33-42, 2004.

[2] W. Dong, L. Sun, and Z. Du, "Design of a precision compliant parallel positioner driven by dual piezoelectric actuators," Sensors and Actuators A: Physical, vol. 135, no. 1, pp. 250-256, March 2007.

[3] Y. Yong, S. Aphale, and S. Moheimani, "Design, identification, and control of a flexure-based xy stage for fast nanoscale positioning," IEEE Trans. on Nanotechnology, vol. 8, no. 1, pp. 46-54, Jan. 2009.

[4] S. Chonan, Z. Jiang, and T. Yamamoto, "Nonlinear hysteresis compensation of piezoelectric ceramic actuators," J. Intell. Mater. Syst. Struct., vol. 7, pp. 150-156, 1996.

[5] M. Al-Janaideh, S. Rakheja, and C. Su, "An analytical generalized prandtl-ishlinskii model inversion for hysteresis compensation in micropositioning control," IEEE/ASME Trans. on Mechatronics, vol. 16, no. 4, pp. 734-744, Aug. 2011.

[6] S. Xiao and Y. Li, "Modeling and high dynamic compensating the ratedependent hysteresis of piezoelectric actuators via a novel modified inverse preisach model," IEEE Trans. on Control Systems Technology, vol. 21 , no. 5, pp. 1549-1557, 2013.

[7] K. Leang and S. Devasia, "Hysteresis, creep, and vibration compensation," IFAC Conf. on Mechatronic Systems, pp. 283-289, 2002.

[8] M. Rakotondrabe, "Bouc-wen modeling and inverse multiplicative structure to compensate hysteresis nonlinearity in piezoelectric actuators," IEEE Trans. on Automation Science and Engineering, vol. 8 , no. 2, pp. 428-431, Apr. 2011.

[9] M. Rakotondrabe, Y. haddab, and P. Lutz, "Quadrilateral modelling and robust control of a nonlinear piezoelectric cantilever," IEEE Trans. on Control Systems Technology, vol. 17, pp. 528-539, 2009.

[10] S. Devasia, E. Eleftheriou, and R. Moheimani, "A survey of control issues in nanopositioning," IEEE Trans. on Control Systems Technology, vol. 15 , no. 15 , pp. 802-823, 2007.

[11] B. Mokaberi and A. A. G. Requicha, "Compensation of scanner creep and hysteresis for afm nanomanipulation," IEEE Trans. on Automation Science and Engineering, vol. 5, no. 2, pp. 197-208, Apr. 2008.

[12] T. Low and W. Guo, "Modeling of a three-layer piezoelectric bimorph beam with hysteresis," J. Microelectromech. Syst., vol. 4, no. 4, p. 230237, Dec. 1995.

[13] E. Grasso, N. Totaro, H. Janocha, and D. Naso, "Piezoelectric self sensing actuators for high voltage excitation," Smart Materials and Structures, vol. 22, no. 6, p. 065018, 2013.

[14] Q. Xu, "A new method of force estimation in piezoelectric cantileverbased microgripper," IEEE/ASME Int. Conf. on Advanced Intelligent Mechatronics (AIM), pp. 574-579, July 11-14 2012.

[15] A. Reddy, N. Maheshwari, D. Sahu, and G. Ananthasuresh, "Miniature compliant grippers with vision-based force sensing," IEEE Trans. on Robotics, vol. 26, no. 5, pp. 867-877, Oct 2010.

[16] B. Komati, J. Agnus, C. Clévy, and P. Lutz, "Prototyping of a highly performant and integrated piezoresistive force sensor for microscale applications," J. Micromech. Microeng., vol. 24, no. 3, p. 035018, March 2014.

[17] H. Kazerooni, T. Sheridan, and P. Houpt, "Robust compliant motion for manipulators, parts i and ii," IEEE J. of Robotics and Automation, vol. 2, no. 2, pp. 83 - 105, June 1986.

[18] R. Ballas, Piezoelectric Multilayer Beam Bending Actuators: Static and Dynamic Behavior and Aspects of Sensor Integration. Springer, 2007.

[19] M. Ismail, F. Ikhouane, and J. Rodellar, "The hysteresis bouc-wen model, a survey," Archives of Computational Methods in Engineering, vol. 16, no. 2, pp. 161-188, 2009. 